\title{
Atorvastatin improves coronary flow and endothelial function in patients with coronary slow flow
}

\author{
HONGMEI NIU ${ }^{1}$, ZHENZHEN WEI $^{2}$, YANLING ZHANG ${ }^{2}$, JIAN HE $^{3}$ and DANYAN JIA ${ }^{4}$ \\ ${ }^{1}$ Department of Cardiovascular Medicine, Shandong Provincial Third Hospital; \\ ${ }^{2}$ Department of Cardiovascular Medicine; ${ }^{3}$ Digestive Disease Department of Internal Medicine, \\ The First People's Hospital of Jinan; ${ }^{4}$ Jinan First Aid Center, Jinan, Shandong 250000, P.R. China
}

Received July 13, 2017; Accepted October 31, 2017

DOI: $10.3892 /$ etm.2017.5484

\begin{abstract}
The underlying mechanisms behind the effect of atorvastatin on patients with coronary slow flow (CSF) remain largely unknown. To investigate the possible underlying molecular mechanisms 108 patients were divided into atorvastatin group and control group. Coronary flow was quantified according to corrected TIMI frame count (CTFC). Serum high sensitivity C-reactive protein (hs-CRP), lipids, ET-1, interleukin (IL)-6, NO, circulating endothelial progenitor cell (cEPC) count, adhesion, migration and proliferation were measured in pretreatment and post-treatment. After respective treatment, the atorvastatin group had significantly decreased levels of TC, TG, LDL-C, hs-CRP, ET-1 and IL- 6 and increased NO compared to the control group. The atorvastatin group had a more significant improvement of CTFC, effective rate, cEPC number, EPC adhesion, migration and proliferation compared to the control group. In conclusion, atorvastatin can be used in treatment of CSF by suppressing inflammation and improving endothelial function.
\end{abstract}

\section{Introduction}

Coronary slow flow (CSF) is a separate clinical entity characterized by delayed coronary opacification without obstructive coronary artery disease $(1,2)$. It was first reported by Tambe et al (3) in 1972 and often associated with chest pain. There is evidence that CSF is linked to several clinical manifestations, such as myocardial ischemia, life-threatening arrhythmias and sudden cardiac death (4-6). Previous studies have demonstrated that CSF occurrence is associated with causes such as inflammation, small vessel disease, endothelial dysfunction and impaired glucose tolerance (7). However, the

Correspondence to: Dr Hongmei Niu, Department of Cardiovascular Medicine, Shandong Provincial Third Hospital, 12 Wuyingshan Road, Jinan, Shandong 250000, P.R. China

E-mail: niuhongmei1234@163.com

Key words: coronary flow, chest pain, inflammation, ET-1, circulating endothelial progenitor cells pathologic mechanism of CSF remains incompletely understood.

Atorvastatin is a member of the statins and usually used as a lipid-lowering agent. Several studies have reported the beneficial effect of atorvastatin for the treatment of dyslipidemia and the prevention of cardiovascular disease $(8,9)$. Its impact on SCF has started to be unveiled. Increasing studies suggest that a long-term therapy of atorvastatin could improve the coronary artery blood flow and coronary flow reserve (CFR) in patients with CSF $(10,11)$. Moreover, Caliskan et al (10) found that short-term lipid-lowering therapy (atorvastatin) improved CFR and coronary microvascular functioning. However, the underlying mechanisms behind the effect of atorvastatin on CFR in patients with SCF remain largely unknown.

To address this issue, we conducted a prospective randomized study on 108 patients with CSF. These patients were randomly divided into two groups: Atorvastatin group and control group. The control group received routine treatment while the atorvastatin group received routine treatment and atorvastatin for 6 months. Clinical efficacy of the two groups were compared. Moreover, serum high sensitivity C-reactive protein (hs-CRP), lipids, endothelin (ET)-1, interleukin (IL)-6, $\mathrm{NO}$, circulating endothelial progenitor cells (cEPCs) count, adhesion, migration and proliferation were measured in patients of the two groups pre-treatment and post-treatment. This study provides added evidence for clinical use of atorvastatin in treatment of CSF and more insights into the underlying pathological mechanisms of CSF.

\section{Materials and methods}

Subjects. This study included 108 patients with CSF, who were admitted in cardiovascular internal medicine department of Shandong Jiaotong Hospital between June 2012 and June 2015. These patients were diagnosed with CSF using coronary angiography (CAG). All participants met the following inclusion criteria: No history of myocardial infarction; no history of coronary interventional surgery; left ventricular ejection fraction $>55 \%$; troponin $(\mathrm{cTnI})<0.01 \mu \mathrm{g} / \mathrm{l}$. The exclusion criteria were: Acute myocardial infarction; liver or kidney dysfunction; atorvastatin allergy; cardiomyopathy; valvular heart disease; hypertensive heart disease; congenital heart disease; 
coronary stenosis or dilation, coronary spasm determined by coronary angiography.

These patients were randomly divided into two groups by the random number generated by computer: Atorvastatin group $(n=54)$ and control group $(n=54)$. The atorvastatin group orally received $40 \mathrm{mg}$ atorvastatin calcium daily (Pfizer Ireland Pharmaceuticals, Ringaskiddy, Ireland), $100 \mathrm{mg}$ aspirin daily (Bayer HealthCare Pharmaceuticals Corporation, Berlin, Germany) and $20 \mathrm{mg}$ isosorbide dinitrate (Livzon Pharmaceutical Factory, Lijiang, China) twice a day for 6 months.

The control group received routine treatment: $100 \mathrm{mg}$ aspirin daily (Bayer HealthCare Pharmaceuticals Corporation) and $20 \mathrm{mg}$ isosorbide dinitrate (Livzon Pharmaceutical Factory) twice a day for 6 months. All these patients provided written informed consent. The study was approved by the Ethics Committee of Shandong Jiaotong Hospital.

Coronary angiography. Coronary angiography was conducted in all patients using the standard Judkins technique. The coronary angiogram was analyzed in a blinded manner by two experienced independent doctors. Slow flow was evaluated using the corrected thrombolysis in myocardial infarction (TIMI) frame count (TFC). Briefly, the first frame was defined as the first frame in which the concentrated dye extended across the entire width of the origin of the artery, touching both borders of the artery lumen. The last frame was defined as the frame in which the contrasted dye first enters the end-point branch off the target artery.

The distal end was defined as the most distal branch of the left anterior descending (LAD) coronary artery and the left circumflex artery (LCX) and the first branch of the posterolateral artery for the right coronary artery (RCA). The anatomic landmark of the anterior descending artery was defined as the distal apical bifurcation. The anatomic landmark of the circumflex artery was the most distal bifurcation of the trunk or branch. The LAD artery was usually longer than other major coronary arteries. Corrected TIMI frame count $($ CTFC) $=$ TIMI frame count/1.7 (12). CSF was defined as no coronary artery stenosis or obstruction was observed and one or more of the three main coronary arteries had CTFC >27 (13).

Measurement of serum hs-CRP, lipids and ET-1, IL-6 and NO. Blood samples were extracted from these patients after overnight fasting one day before and one day after the treatment. These samples were centrifuged at at $1,800 \mathrm{x} \mathrm{g}$ for $15 \mathrm{~min}$. The supernatant was collected in a centrifuge tube and stored at $-80^{\circ} \mathrm{C}$. ET-1, IL- 6 and hs-CRP in serum were measured using ELISA kits (Nanjing Jiancheng Bioengineering Institute, Nanjing, China). Each sample was measured three times. NO level was measured by using a colorimetric detection kit (Nanjing Jiancheng Bioengineering Institute). Total cholesterol (TC), high density lipoprotein cholesterol (HDL-C), low density lipoprotein cholesterol (LDL-C) and triglycerides (TG) were evaluated using an automatic biochemical analyzer (Hitachi 7180; Hitachi Ltd., Ibaraki, Japan).

Evaluation of clinical outcome. Clinical efficacy was evaluated according to the chest pain and angina pectoris syndromes of
Table I. Demographic data of patients

\begin{tabular}{lcc}
\hline Patient data & $\begin{array}{c}\text { Atorvastatin group } \\
(\mathrm{n}=54)\end{array}$ & $\begin{array}{c}\text { Control group } \\
(\mathrm{n}=54)\end{array}$ \\
\hline Age (years) & $55.86 \pm 7.92$ & $56.63 \pm 6.53$ \\
Sex (male/female) & $35 / 19$ & $33 / 21$ \\
BMI $\left(\mathrm{kg} / \mathrm{m}^{3}\right)$ & $26.46 \pm 4.27^{\mathrm{a}}$ & $27.15 \pm 3.58^{\mathrm{a}}$ \\
Smoking $(\mathrm{n} / \%)$ & $25(46.30)$ & $26(48.15)$ \\
Hypertension $(\mathrm{n} / \%)$ & $22(40.74)$ & $23(42.59)$ \\
Diabetes $(\mathrm{n} / \%)$ & $10(18.52)$ & $10(18.52)$ \\
Hyperlipidemia $(\mathrm{n} / \%)$ & $26(48.15)$ & $27(50.00)$ \\
Family history of & $17(31.48)$ & $16(29.63)$ \\
coronary heart & & \\
disease $(\mathrm{n} / \%)$ & & \\
\hline
\end{tabular}

${ }^{\mathrm{a}} \mathrm{P}<0.01$ compared to the control group.

patients. Markedly effective: Almost no chest pain or angina pectoris was detected and the frequency and the duration of chest pain or angina pectoris was decreased by more than $80 \%$. Effective: The frequency and the duration of chest pain or angina pectoris was decreased by $50-80 \%$. Valid: The frequency and the duration of chest pain or angina pectoris was decreased by less than $50 \%$ or the syndromes were not changed or deteriorated.

cEPCs isolation and culture. An aliquot of $10 \mathrm{ml}$ peripheral blood was extracted from all patients after overnight fasting. After adding heparin for anticoagulation, the blood sample was mixed with Hanks solution (1:1) followed by addition of mononuclear cell separation medium. Mononuclear cells were retrieved after centrifugation at $900 \mathrm{x}$ g for $20 \mathrm{~min}$. The cell suspension was seeded onto the cell slides coating human fibronectin protein (HFN; Santa Cruz Biotechnology, Santa Cruz, CA, USA) and cultured in M199 medium (Gibco, Grand Island, NY, USA) supplemented with penicillin (100 U/ml), streptomycin $(100 \mathrm{U} / \mathrm{ml})$ and $20 \%$ fetal bovine serum (FBS; Hyclone Laboratories, Logan, UT, USA).

After washing with PBS, the non-adherent cells were deleted. The cell slides were incubated in DiI-Ac-LDL $(24 \mu \mathrm{g} / \mathrm{ml})$ at $37^{\circ} \mathrm{C}$ for $1 \mathrm{~h}$. The staining was observed under an inverted fluorescence microscope (Nikon, Tokyo, Japan) for DiI-Ac-LDL intake. The cell slides were fixed in $2 \%$ paraformaldehyde for $10 \mathrm{~min}$. After PBS washing, the cell slides were incubated with FITC-UEA-I $(10 \mu \mathrm{g} / \mathrm{ml}) 37^{\circ} \mathrm{C}$ for $1 \mathrm{~h}$. DiI-Ac-LDL and FITC-UEA-I double positive cells were identified using a confocal microscope (Bio-Rad Laboratories, Hercules, CA, USA). Five observation fields were randomly selected under an inverted fluorescence microscope for calculation of cEPCs count.

Evaluation of cEPCs adhesion. After digestion with $0.25 \%$ trypsin, the cEPCs were suspended in $500 \mu \mathrm{l}$ medium. The cell suspension was embedded in HFN-coated cell culture plates which was then placed in an incubator for $30 \mathrm{~min}$. Three randomly chosen fields were observed under a microscope to observe the adherent cells. 
Table II. Measurement of serum hs-CRP, lipids, ET-1, IL-6 and NO.

\begin{tabular}{lcccc}
\hline & \multicolumn{2}{c}{ Atorvastatin group } & \multicolumn{2}{c}{ Control group } \\
\cline { 2 - 4 } Parameters & Pretreatment & Post-treatment & Pretreatment & \multirow{2}{*}{ Post-treatment } \\
\hline TC $(\mathrm{mmol} / \mathrm{l})$ & $5.17 \pm 0.73$ & $3.51 \pm 0.65^{\mathrm{a}, \mathrm{b}}$ & $5.21 \pm 0.80$ & $4.05 \pm 0.64^{\mathrm{a}}$ \\
TG $(\mathrm{mmol} / \mathrm{l})$ & $1.78 \pm 0.62$ & $0.67 \pm 0.58^{\mathrm{a}, \mathrm{b}}$ & $1.74 \pm 0.59$ & $1.27 \pm 0.50^{\mathrm{a}}$ \\
LDL-C (mmol/l) & $3.42 \pm 0.49$ & $1.98 \pm 0.61^{\mathrm{a}, \mathrm{b}}$ & $3.41 \pm 0.55$ & $2.64 \pm 0.59^{\mathrm{a}}$ \\
HDL-C $(\mathrm{mmol} / \mathrm{l})$ & $1.69 \pm 0.81$ & $1.58 \pm 0.70$ & $1.71 \pm 0.77$ & $1.54 \pm 0.75$ \\
Hs-CRP $(\mathrm{mg} / \mathrm{l})$ & $4.32 \pm 1.61$ & $1.08 \pm 0.63^{\mathrm{a}, \mathrm{b}}$ & $4.28 \pm 1.59$ & $2.25 \pm 1.21^{\mathrm{a}}$ \\
ET-1 $(\mathrm{ng} / \mathrm{l})$ & $119.23 \pm 21.75$ & $78.52 \pm 18.26^{\mathrm{a}, \mathrm{b}}$ & $121.96 \pm 22.24$ & $109.78 \pm 19.84^{\mathrm{a}}$ \\
IL-6 $(\mathrm{ng} / \mathrm{l})$ & $171.35 \pm 58.19$ & $104.38 \pm 46.75^{\mathrm{a}, \mathrm{b}}$ & $169.63 \pm 59.84$ & $147.79 \pm 50.67^{\mathrm{a}}$ \\
NO $(\mu \mathrm{mol} / \mathrm{l})$ & $28.98 \pm 14.08$ & $42.62 \pm 16.38^{\mathrm{a}, \mathrm{b}}$ & $28.54 \pm 13.53$ & $38.70 \pm 16.26^{\mathrm{a}}$ \\
\hline
\end{tabular}

${ }^{a} \mathrm{P}<0.05$ compared to pretreatment results of the same group; ${ }^{\mathrm{b}} \mathrm{P}<0.05$ compared to the post-treatment results of the control group. TC, total cholesterol; TG, triglycerides, LDL-C, low density lipoprotein cholesterol; HDL-C, high density lipoprotein cholesterol; Hs-CRP, high sensitivity C-reactive protein; IL-6, interleukin-6.

Evaluation of cEPC migration. EPC migration was assessed using Falcon HTS FluoroBlok 24-well inserts with $8-\mu \mathrm{m}$ pores (BD Biosciences, San Jose, CA, USA). An aliquot of $100 \mu \mathrm{l}$ of EPCs suspension was added in the upper chamber with $600 \mu 1$ M199 medium. These 24-well inserts were cultured in an incubator for $24 \mathrm{~h}$. The cells on the upper surface of the membrane were removed by PBS washing. The migrated cells on the bottom surface were fixed in $4 \%$ paraformaldehyde and stained with Giemsa. Three randomly chosen fields were observed under a microscope to calculate the migrated cells.

Methyl thiazolyl tetrazolium assay (MTT). cEPC proliferation was evaluated using MTT assay. Briefly, EPC suspension (100 $\mu \mathrm{l} /$ well) was seeded into 96 -well plates. An aliquot of $20 \mu \mathrm{l}$ MTT (5 g/l; Sigma-Aldrich, St. Louis, MO, USA) was added into each well. The cell plates were cultured in an incubator for $4 \mathrm{~h}$. After discarding the supernatant, dimethyl sulphoxide (DMSO; Sigma-Aldrich, USA) was added into each well (150 $\mu \mathrm{l} /$ well). Finally, the formation of the formazan product was measured colorimetrically at $490 \mathrm{~nm}$.

Statistical analysis. Statistical analysis was performed using SPSS software version 18.0 (SPSS Inc., Chicago, IL, USA). Quantitative data (mean \pm standard deviation) between groups were compared using Student' t-test. Categorical data between groups were compared using Chi-square test. $\mathrm{P}<0.05$ was considered to indicate a statistically significant difference.

\section{Results}

Serum lipids, hs-CRP, ET-1, IL-6 and NO. The atorvastatin group and the control group were not significantly different for age, gender, BMI, smoking, hypertension, diabetes, hyperlipidemia and family history of coronary heart disease (Table I).

In order to evaluate the effect of atorvastatin on serum lipids and inflammation-related factors $(14,15)$, this study measured TC, TG, LDL-C, HDL-C, hs-CRP, ET-1, IL-6 and $\mathrm{NO}$ in the two groups (Table II). Before the treatment, serum
Table III. TIMI frame count of patients.

\begin{tabular}{llllll}
\hline & \multicolumn{2}{c}{ Atorvastatin group } & & \multicolumn{2}{c}{ Control group } \\
\cline { 2 - 3 } \cline { 5 - 6 } CTFC & Pretreatment & Post-treatment & Pretreatment & Post-treatment \\
\hline LAD & $46.63 \pm 14.12$ & $28.64 \pm 10.46^{\mathrm{a}, \mathrm{b}}$ & $47.26 \pm 11.12$ & $34.37 \pm 10.29^{\mathrm{a}}$ \\
LCX & $49.22 \pm 13.17$ & $30.56 \pm 6.74^{\mathrm{a}, \mathrm{b}}$ & $52.83 \pm 9.06$ & $41.21 \pm 13.61^{\mathrm{a}}$ \\
RCA & $52.93 \pm 13.82$ & $33.80 \pm 14.72^{\mathrm{a}, \mathrm{b}}$ & $51.48 \pm 14.07$ & $39.96 \pm 11.62^{\mathrm{a}}$ \\
TIMI & $49.86 \pm 12.64$ & $30.93 \pm 11.27^{\mathrm{a}, \mathrm{b}}$ & $51.05 \pm 11.07$ & $38.48 \pm 10.84^{\mathrm{a}}$ \\
frame & & & & \\
count & & & & \\
\hline
\end{tabular}

TIMI, thrombolysis in myocardial infarction; CTFC, corrected TIMI frame count; LAD, left anterior descending coronary artery; LCX, left circumflex artery; RCA, right coronary artery. ${ }^{\mathrm{a}} \mathrm{P}<0.05$ compared to the pretreatment result of the same group; ${ }^{b} \mathrm{P}<0.05$ compared to the post-treatment result of the control group.

levels of TC, TG, LDL-C, HDL-C, hs-CRP, ET-1, IL-6 and $\mathrm{NO}$ were not significantly different between the atorvastatin group and the control group $(\mathrm{P}>0.05)$. After respective treatment, TC, TG, LDL-C, hs-CRP, ET-1 and IL-6 were obviously decreased, while NO was markedly increased compared to the pretreatment results in both the atorvastatin group and the control group $(\mathrm{P}<0.05)$. Moreover, the atorvastatin group had significantly decreased post-treatment TC, TG, LDL-C, hs-CRP, ET-1 and IL-6 and increased NO compared to the control group $(\mathrm{P}<0.05)$. These findings suggest that the atorvastatin treatment could lower serum lipids.

TIMI frame count and clinical efficacy. As shown in Table III, the atorvastatin group and the control group were not significantly different in pretreatment CTFC ( $\mathrm{P}>0.05)$. Both groups had significantly decreased post-treatment CTFC compared to the pretreatment CTFC $(\mathrm{P}<0.05)$. Moreover, the post-treatment CTFC of the atorvastatin group was further decreased than that of the control group $(\mathrm{P}<0.05)$. These observations indicate that both routine therapy and atorvastatin therapy could improve CTFC in CSF patients. The atorvastatin group 
Table IV. Clinical efficacy.

\begin{tabular}{lcccc}
\hline Group & $\begin{array}{c}\text { Markedly } \\
\text { effective }\end{array}$ & Effective & Valid & $\begin{array}{c}\text { Effective rate } \\
\text { (markedly effective + } \\
\text { effective, \%) }\end{array}$ \\
\hline $\begin{array}{l}\text { Atorvastatin } \\
\text { group } \\
\begin{array}{l}\text { Control } \\
\text { group }\end{array}\end{array}$ & 35 & 11 & 8 & $85.19^{\mathrm{a}}$ \\
\hline
\end{tabular}

${ }^{\mathrm{a}} \mathrm{P}<0.01$, compared to the control group.

Table V. EPCs count, adhesion, migration and proliferation.

\begin{tabular}{|c|c|c|c|c|}
\hline \multirow[b]{2}{*}{ cEPCs } & \multicolumn{2}{|c|}{ Atorvastatin group } & \multicolumn{2}{|c|}{ Control group } \\
\hline & Pre-treatment & $\begin{array}{c}\text { Post- } \\
\text { treatment }\end{array}$ & Pre-treatment & $\begin{array}{c}\text { Post- } \\
\text { treatment }\end{array}$ \\
\hline $\begin{array}{l}\text { cEPCs } \\
\text { count/HP } \\
(\mathrm{x} 200)\end{array}$ & $38.58 \pm 9.12$ & $52.69 \pm 9.46^{\mathrm{a}, \mathrm{b}}$ & $38.97 \pm 8.55$ & $42.37 \pm 10.29$ \\
\hline $\begin{array}{l}\text { Adherent } \\
\text { cEPCs/HP } \\
(\mathrm{x} 200)\end{array}$ & $20.95 \pm 7.13$ & $26.27 \pm 7.21^{a, b}$ & $20.83 \pm 6.55$ & $21.21 \pm 8.65$ \\
\hline $\begin{array}{l}\text { Migrated } \\
\text { cEPCs/HP } \\
(\mathrm{x} 200)\end{array}$ & $10.39 \pm 8.23$ & $16.58 \pm 7.42^{\mathrm{a}, \mathrm{b}}$ & $10.56 \pm 8.27$ & $10.79 \pm 7.64$ \\
\hline $\begin{array}{l}\text { cEPCs } \\
\text { proliferation } \\
\text { (A490) }\end{array}$ & $0.327 \pm 0.043$ & $0.539 \pm 0.037^{\mathrm{a}, \mathrm{b}}$ & $0.328 \pm 0.027$ & $0.352 \pm 0.054$ \\
\hline
\end{tabular}

EPCs, endothelial progenitor cells; cEPCs, circulating endothelial progenitor cells; HP, Helicobacter pylori. ${ }^{a} \mathrm{P}<0.05$ compared to the pretreatment result of the same group; ${ }^{b} \mathrm{P}<0.05$ compared to the posttreatment result of the control group.

had a more significant improvement than the control group. After respective treatment, the effective rate of the atorvastatin group was markedly elevated compared to that of the control group ( $>>0.05$, Table IV). It reveals that the atorvastatin treatment is more effective than the routine therapy in alleviating chest pain or angina pectoris syndromes of patients with CSF.

EPC count, adhesion, migration and proliferation. It has been reported that endothelial dysfunction is an important cause of CSF (16,17). Therefore, the study also compared the EPC count, adhesion, migration and proliferation in the two groups. Table V shows that before the treatment, the two groups were not significantly different with regard to EPC count, adhesion, migration and proliferation $(\mathrm{P}>0.05)$. After respective treatment, the EPC count was significantly increased $(\mathrm{P}<0.05)$ and the EPC adhesion, migration and proliferation were much improved in the atorvastatin group relative to the control group $(\mathrm{P}<0.05)$.

These findings suggest that atorvastatin therapy could increase EPC count and improve cEPCs adhesion, migration and proliferation.

\section{Discussion}

CSF is defined as delayed distal perfusion during coronary angiography with normal coronary arteries (18). This study investigated the impact of atorvastatin on patients with CSF and the underlying mechanisms. It found that compared to the routine treatment, atorvastatin therapy had a better efficacy in lowering TC, TG, LDL-C, hs-CRP, ET-1 and IL-6, increasing NO, improving CTFC and endothelial functioning, alleviating chest pain or angina pectoris syndromes in CSF patients.

Emerging studies have established that atorvastatin therapy could improve coronary artery blood flow and CFR in patients with CSF $(10,11)$. In line with previous finding, the present study found that atorvastatin therapy was superior to the routine treatment in improving CSF and alleviating chest pain or angina pectoris syndromes in patients with CSF.

Nitric oxide is mostly produced by the endothelial isoform (eNOS) in endothelial cells and plays an important part in vascular tone and structure regulation (19) and the regulation of coronary blood flow (20). A rich body of evidence suggest that atorvastatin increases inducible NO synthase $(21,22)$. There is in vivo evidence that atorvastatin increases endothelial cytoprotective NO in diabetic, hypertensive rats (23). Consistent with the previous studies, this study found that atorvastatin therapy led to increased level of NO compared to the routine treatment in patients with CSF. It indicates that the improvement of CSF by atorvastatin therapy flow might be partly due to the increase in the NO level.

It has been demonstrated that atorvastatin therapy could prohibit arterial inflammation as well as various circulating biomarkers, such as hs-CRP (24). van de Ree et al (25) in a prospective double-blind multicenter study found that high dose atorvastatin led to an evident decrease in hs-CRP level in patients with type 2 diabetes. Moreover, increasing studies have demonstrated that atorvastatin could suppress the production of IL-6 (26,27). Likewise, the study confirmed the anti-inflammatory effect of atorvastatin, as evidenced by the fact that the atorvastatin group had markedly decreased levels of hs-CRP and IL-6 compared to the control group. ET-1 is a potent vasoconstrictor produced by vascular endothelial cells. Chang et al (28) provided in vivo evidence that atorvastatin preconditioning decreased the production of ET-1 in a rat model. Similarly, the study found that the ET-1 level was significantly lower in the atorvastatin group than that in the control group. These findings suggest that the atorvastatin therapy is more effective than the routine treatment in inhibiting inflammation and decreasing ET-1 level in patients with CSF. Atorvastatin therapy might improve CSF by suppressing inflammation and decreasing ET-1 level.

Endothelial progenitor cells (EPCs) play an important role in the maintenance of vascular integrity. Minami et al (29) have reported that lipid-lowering therapy with atorvastatin could increase EPC numbers in patients with coronary artery disease.

Moreover, Oikonomou et al (30) revealed that high dose atorvastatin treatment has a great beneficial effect on endothelial function and increased the number of cEPCs in ischemic heart failure patients. In concordance with these observations, the study found that compared to the routine therapy, the atorvastatin therapy has increased EPC count and improved 
cEPC adhesion, migration and proliferation in patients with CSF. It indicates that atorvastatin therapy may be more effective than the routine therapy in improving endothelial function and increasing the number of cEPCs, thereby improving CSF.

The study has some limitations. The number of patients with CSF is limited and the duration of atorvastatin therapy is short. More studies with longer-term atorvastatin use are needed in a larger number of patients with CSF.

The study suggests that atorvastatin therapy may improve CSF and alleviate chest pain or angina pectoris syndromes in patients with CSF by suppressing inflammation, decreasing ET-1, increasing NO and the number of cEPCs and improving endothelial function. These findings would contribute to an improved treatment of CSF. More studies are warranted to validate and extend the findings of the study.

\section{References}

1. Hawkins BM, Stavrakis S, Rousan TA, Abu-Fadel M and Schechter E: Coronary slow flow - prevalence and clinical correlations. Circ J 76: 936-942, 2012.

2. Beltrame JF: Defining the coronary slow flow phenomenon. Circ J 76: 818-820, 2012.

3. Tambe AA, Demany MA, Zimmerman HA and Mascarenhas E: Angina pectoris and slow flow velocity of dye in coronary arteries - a new angiographic finding. Am Heart J 84: 66-71, 1972.

4. Cutri N, Zeitz C, Kucia AM and Beltrame JF: ST/T wave changes during acute coronary syndrome presentation in patients with the coronary slow flow phenomenon. Int J Cardiol 146: 457-458, 2011

5. Horjeti B and Goda A: Acute ischemia manifestation in a patient with coronary slow flow phenomenon. J Electrocardiol 45: 277-279, 2012 .

6. Saya S, Hennebry TA, Lozano P, Lazzara R and Schechter E: Coronary slow flow phenomenon and risk for sudden cardiac death due to ventricular arrhythmias: A case report and review of literature. Clin Cardiol 31: 352-355, 2008.

7. Wang $X$ and Nie SP: The coronary slow flow phenomenon: Characteristics, mechanisms and implications. Cardiovasc Diagn Ther 1: 37-43, 2011.

8. Yoshida H, Shoda T, Yanai H, Ikewaki K, Kurata H, Ito K, Furutani N, Tada N, Witztum JL and Tsimikas S: Effects of pitavastatin and atorvastatin on lipoprotein oxidation biomarkers in patients with dyslipidemia. Atherosclerosis 226: 161-164, 2013.

9. Colhoun HM, Betteridge DJ, Durrington PN, Hitman GA, Neil HA, Livingstone SJ, Thomason MJ, Mackness MI, Charlton-Menys V and Fuller JH; CARDS investigators: Primary prevention of cardiovascular disease with atorvastatin in type 2 diabetes in the Collaborative Atorvastatin Diabetes Study (CARDS): Multicentre randomised placebo-controlled trial. Lancet 364: 685-696, 2004.

10. Caliskan M, Erdogan D, Gullu H, Topcu S, Ciftci O, Yildirir A and Muderrisoglu $\mathrm{H}$ : Effects of atorvastatin on coronary flow reserve in patients with slow coronary flow. Clin Cardiol 30: 475-479, 2017.

11. Baykan AO, Kalkan GY, Sahin DY, Elbasan Z, Gur M, Seker T, Turkoglu $\mathrm{C}$ and Caylı M: Coronary flow velocity reserve in donor artery and myocardial performance index after successful recanalization of chronic total coronary occlusions. J Invasive Cardiol 27: E75-E81, 2015.

12. Gibson CM, Cannon CP, Daley WL, Dodge JT Jr, Alexander B Jr, Marble SJ, McCabe CH, Raymond L, Fortin T, Poole WK, et al: TIMI frame count: A quantitative method of assessing coronary artery flow. Circulation 93: 879-888, 1996.

13. Nie SP, Wang X, Geng LL, Liu BQ, Li J, Qiao Y, Liu XM, Luo TY, Dong JZ, Liu XH, et al: Anatomic properties of coronary arteries are correlated to the corrected thrombolysis in myocardial infarction frame count in the coronary slow flow phenomenon. Coron Artery Dis 23: 174-180, 2012.

14. Sugiyama M, Ohashi M, Takase H, Sato K, Ueda R and Dohi Y: Effects of atorvastatin on inflammation and oxidative stress. Heart Vessels 20: 133-136, 2005.
15. Bielecka-Dabrowa A, Mikhailidis DP, Rizzo M, von Haehling S, Rysz J and Banach M: The influence of atorvastatin on parameters of inflammation left ventricular function, hospitalizations and mortality in patients with dilated cardiomyopathy - 5-year follow-up. Lipids Health Dis 12: 47, 2013.

16. Kopetz V, Kennedy J, Heresztyn T, Stafford I, Willoughby SR and Beltrame JF: Endothelial function, oxidative stress and inflammatory studies in chronic coronary slow flow phenomenon patients. Cardiology 121: 197-203, 2012.

17. Yoon HJ, Jeong MH, Cho SH, Kim KH, Lee MG, Park KH, Sim DS, Yoon NS, Hong YJ, Kim JH, et al: Endothelial dysfunction and increased carotid intima-media thickness in the patients with slow coronary flow. J Korean Med Sci 27: 614-618, 2012.

18. Beltrame JF, Limaye SB, Wuttke RD and Horowitz JD: Coronary hemodynamic and metabolic studies of the coronary slow flow phenomenon. Am Heart J 146: 84-90, 2003.

19. Desjardins F and Balligand JL: Nitric oxide-dependent endothelial function and cardiovascular disease. Acta Clin Belg 61: 326-334, 2006.

20. Toda N, Tanabe S and Nakanishi S: Nitric oxide-mediated coronary flow regulation in patients with coronary artery disease: Recent advances. Int J Angiol 20: 121-134, 2011.

21. Atar S, Ye Y, Lin Y, Freeberg SY, Nishi SP, Rosanio S, Huang MH, Uretsky BF, Perez-Polo JR and Birnbaum Y: Atorvastatin-induced cardioprotection is mediated by increasing inducible nitric oxide synthase and consequent S-nitrosylation of cyclooxygenase-2. Am J Physiol Heart Circ Physiol 290: H1960-H1968, 2006.

22. Rajamannan NM, Subramaniam M, Stock SR, Stone NJ, Springett M, Ignatiev KI, McConnell JP, Singh RJ, Bonow RO and Spelsberg TC: Atorvastatin inhibits calcification and enhances nitric oxide synthase production in the hypercholesterolaemic aortic valve. Heart 91: 806-810, 2005.

23. Mason RP, Corbalan JJ, Jacob RF, Dawoud H and Malinski T: Atorvastatin enhanced nitric oxide release and reduced blood pressure, nitroxidative stress and rantes levels in hypertensive rats with diabetes. J Physiol Pharmacol 66: 65-72, 2015.

24. Wu YW, Kao HL, Huang CL, Chen MF, Lin LY, Wang YC, Lin YH, Lin HJ, Tzen KY, Yen RF, et al: The effects of 3-month atorvastatin therapy on arterial inflammation, calcification, abdominal adipose tissue and circulating biomarkers. Eur J Nucl Med Mol Imaging 39: 399-407, 2012.

25. van de Ree MA, Huisman MV, Princen HMG, Meinders AE and Kluft C; DALI-Study Group: Strong decrease of high sensitivity C-reactive protein with high-dose atorvastatin in patients with type 2 diabetes mellitus. Atherosclerosis 166: 129-135, 2003.

26. Zhang YY, Fan YC, Wang M, Wang D and Li XH: Atorvastatin attenuates the production of IL-1 $\beta$, IL- 6 , and TNF- $\alpha$ in the hippocampus of an amyloid $\beta 1-42$-induced rat model of Alzheimer's disease. Clin Interv Aging 8: 103-110, 2013.

27. Wang H, Cui XX, Goodin S, Ding N, Van Doren J, Du Z, Huang MT, Liu Y, Cheng X, Dipaola RS, et al: Inhibition of IL-6 expression in $\mathrm{LNCaP}$ prostate cancer cells by a combination of atorvastatin and celecoxib. Oncol Rep 31: 835-841, 2014.

28. Chang CZ, Wu SC, Lin CL, Hwang SL, Howng SL and Kwan AL: Atorvastatin preconditioning attenuates the production of endothelin-1 and prevents experimental vasospasm in rats. Acta Neurochir (Wien) 152: 1399-1406, 2010.

29. Minami Y, Satoh M, Maesawa C, Takahashi Y, Tabuchi T, Itoh T and Nakamura M: Effect of atorvastatin on microRNA 221/222 expression in endothelial progenitor cells obtained from patients with coronary artery disease. Eur J Clin Invest 39: 359-367, 2009

30. Oikonomou E, Tousoulis D, Siasos G, Zaromitidou M, Chrysohoou C, Kokkou E, Tourikis P, Mazaris S, Konsola T and Stefanadis C: The impact of high dose atorvastatin treatment on endothelial progenitor cells, vascular function and inflammatory status in ischemic heart failure. Eur Heart J 34 (Suppl 1): P5724-P5724, 2013.

This work is licensed under a Creative Commons Attribution-NonCommercial-NoDerivatives 4.0 International (CC BY-NC-ND 4.0) License. 